\title{
On Expansion in the Width for Domain Walls *
}

\author{
by \\ H. Arodź \\ Institute of Physics, Jagellonian University, Cracow ${ }^{\dagger}$
}

\begin{abstract}
The well-known idea to construct domain wall type solutions of field equations by means of an expansion in the width of the domain wall is reexamined. We observe that the problem involves singular perturbations. HilbertChapman-Enskog method is used to construct a consistent perturbative expansion. We obtain the solutions to the second order in the width without introducing an effective action for the domain wall. We find that zeros of the scalar field in general do not lie on a Nambu-Goto trajectory. As examples we consider cylindrical and spherical domain walls. We find that the spherical domain wall, in contradistinction to the cylindrical one, shows an effective rigidity.
\end{abstract}

January 1995

TPJU-2/95

\footnotetext{
*Paper supported in part by the grant KBN 2 P302 04905.

${ }^{\dagger}$ Address: Reymonta 4, 30-059 Cracow, Poland.

${ }^{\ddagger}$ E-mail: ufarodz@ztc386a.if.uj.edu.pl
} 


\section{Introduction}

Dynamics of topological defects is to be deduced from underlying nonlinear equations describing evolution of fields the defect is composed of. In general, it is a formidable task and one is forced to resort to approximate analytical solutions or to numerical solutions. The results are of great interest for particle physics (e.g. for dynamics of a flux-tube in QCD [1]), for fieldtheoretical cosmology (e.g. cosmic strings [2]), and for condensed matter physics (e.g. domain walls in magnetics [3], vortices in superconductors [4] or in superliquid Helium [5], defects in liquid crystals [6]).

In particular, in the context of relativistic field theoretical models there is a long-standing, interesting and difficult problem how to calculate evolution of vortices and of domain walls. Theoretical descriptions of vortices and domain walls show many similarities, and the two kinds of objects can be treated by essentially identical methods. The problem has become even more interesting after the seminal paper [7] has appeared, in which it has been pointed out that the vortex dynamics can approximately be described in terms of a string model. Apart from purely numerical computations, see e.g. [8, 9, 10], the predominant approach is the effective action method, started in the paper [11] and pursued in numerous papers till nowadays, see e.g. [12, 13, 14, 15, 16, 17, 18, 19]. The idea is to reduce the complicated field-theoretical dynamics to much simpler dynamics of a string or a membrane by regarding some degrees of freedom as inessential. In the first step, these degrees of freedom are eliminated by the assumption that they have definite values determined from a conditional stationary action principle for the original field-theoretical action. The condition usually adopted is that zeros of the scalar field have apriori fixed, arbitrary trajectory in Minkowski space-time (identified with the world-volume of the membrane). Inserting these conditional solutions into the original action one obtains the effective action for the membrane. In the second step one has to find solutions of the equations of motion for the membrane, derived from the action principle for the effective action. Let us stress that in this approach calculation of the effective action is the necessary intermediate step. Without it, one has only the whole set of conditional solutions which exist for all trajectories of the membrane, including unphysical ones. It is just the role of the second step to pick physical trajectories for the membrane. See the paper [15] for an explicit formulation and [20] for a discussion of this method. 
Another group of approaches aims at deriving directly from the original field equations dynamical equations for certain characteristics of the topological defect, e.g. for an average radius in the case of a ring-like vortex [21], or position of zeros of the Higgs field [22]. In these approaches one does not have to calculate the effective action as the necessary intermediate step. To this group belongs also the approach based on a polynomial approximation to the fields constituting the defect 25 - in this approach also a width of the domain wall is a dynamical variable.

In the present paper we would like to discuss one more method of calculating the evolution of domain walls or vortices directly from the original field equations: an expansion in the width of the domain wall or a vortex. This method has been tried in many papers, e.g. in [11]-19], always in conjunction with the effective action method. In the present paper we show that the expansion in the width itself, when appropriately carried out, is sufficient to determine evolution of the defect. No effective action is necessary. We will describe our formulation of this method in the case of the domain walls. Analogous calculation for vortices will be presented as a separate paper.

Our main steps are moreless the same as in the previous papers: we introduce a special coordinate system co-moving with the domain wall and we formally expand the sought for solution of the field equation in the positive powers of the width of the domain wall. (The actual expansion parameters are dimensionless ratios of the width with curvature radia of the domain wall.) The first new feature specific for our formulation is that we do not tie the co-moving coordinate system with position of the zeros of the scalar field. It is tied to a Nambu-Goto membrane - this fact follows from the field equation as a consistency condition. However this membrane in general is not the locus of the zeros of the field. The position of the zeros is determined afterwards, from the explicitely constructed approximate solution of the field equation.

Second, we observe that the expansion in the width is an example of so called singular perturbations [24]. In such case one has to take into account certain consistency conditions for the perturbative expansion. Using HilbertChapman-Enskog method for constructing perturbative expansion in the case of singular perturbations, see 25 for a lucid description of it, we obtain the domain wall solution directly by approximate solving the original field equation, without any need to introduce the effective action.

The plan of our paper is as follows. In Section 2 we introduce the co- 
moving coordinates and we present the field equation. This section is included mainly for convenience of the reader. In Section 3 we apply the Hilbert-Chapman-Enskog method to calculate the domain wall solution in the perturbative expansion in positive powers of the width. In Section 4 we present simple examples: solutions describing cylindrical and spherical domain walls. Section 5 contains ending remarks.

\section{Transformation to the co-moving coordi- nates}

We shall consider domain walls in a well-known model defined by the following Lagrangian

$$
L=-\frac{1}{2} \eta_{\mu \nu} \partial^{\mu} \Phi \partial^{\nu} \Phi-\frac{\lambda}{2}\left(\Phi^{2}-\frac{M^{2}}{4 \lambda}\right)^{2},
$$

where $\left(\eta_{\mu \nu}\right)=\operatorname{diag}(-1,1,1,1)$, and $\lambda, M$ are positive constants. The corresponding field equation for the scalar field $\Phi$ has the form

$$
\partial_{\mu} \partial^{\mu} \Phi-2 \lambda\left(\Phi^{2}-\frac{M^{2}}{4 \lambda}\right) \Phi=0
$$

There are two vacuum values of the field $\Phi$ equal to $\pm \Phi_{0}$, where $\Phi_{0} \equiv$ $M / 2 \sqrt{\lambda}$. The domain wall is given by the field $\Phi$ smoothly interpolating between the two vacua. At each instant of time the field $\Phi$ vanishes somewhere inside the domain wall. We assume that the locus of these zeros is a smooth surface $S$. We shall call it the core of the domain wall. Well-known example of the domain wall, with the core given by the $\left(x^{1}, x^{2}\right)$ plane, is given by the following exact, static solution of Eq.(2)

$$
\Phi=\Phi_{0} \tanh \left(\frac{x^{3}}{2 l_{0}}\right)
$$

where $l_{0} \equiv M^{-1}$. The width of this domain wall is of the order $l_{0}$, and energy density is exponentially localised around the $\left(x^{1}, x^{2}\right)$ plane.

For a generic domain wall, we parametrise the world-volume $\Sigma$ of the core (a 3-dimensional manifold embedded in Minkowski space-time, whose time 
slices coincide with $S$ ) as follows

$$
\left(X^{\mu}\right)\left(\tau, \sigma^{1}, \sigma^{2}\right)=\left(\begin{array}{c}
\tau \\
X^{1}\left(\tau, \sigma^{1}, \sigma^{2}\right) \\
X^{2}\left(\tau, \sigma^{1}, \sigma^{2}\right) \\
X^{3}\left(\tau, \sigma^{1}, \sigma^{2}\right)
\end{array}\right),
$$

where $\tau$ coincides with the laboratory frame time $x^{0}$, and $\sigma^{1}, \sigma^{2}$ parametrise the core $S$ at each instant of time.

We shall also need another surface $\tilde{S}$ attached to the domain wall. In general $\tilde{S} \neq S$. In the next section we shall obtain a Nambu-Goto equation for $\tilde{S}$, so this surface can be regarded as the Nambu-Goto type relativistic membrane. The surface $\tilde{S}$ is related to the core $S$ by a simple formula (51) derived in Section 4. In the following we shall call $\tilde{S}$ the membrane comoving with the domain wall. Let us stress that the membrane is merely an auxilliary mathematical constructs - the physical object is the domain wall.

The world-volume of the membrane will be denoted by $\tilde{\Sigma}$, and we shall parametrise it as follows

$$
\left(Y^{\mu}\right)\left(\tau, \sigma^{1}, \sigma^{2}\right)=\left(\begin{array}{c}
\tau \\
Y^{1}\left(\tau, \sigma^{1}, \sigma^{2}\right) \\
Y^{2}\left(\tau, \sigma^{1}, \sigma^{2}\right) \\
Y^{3}\left(\tau, \sigma^{1}, \sigma^{2}\right)
\end{array}\right)
$$

where $\tau$ coincides with the laboratory frame time $x^{0}$. The parameters $\sigma^{1}, \sigma^{2}$ in formula (5) are in principle independent of the ones in formula (4). We use the same notation for convenience, and also in anticipation of a one-to-one correspondence between $S$ and $\tilde{S}$ given by formula (51).

As usual, we introduce a special coordinate system $\left(\tau, \sigma^{1}, \sigma^{2}, \xi\right)$ co-moving with the domain wall. These coordinates are defined by the following formula

$$
x^{\mu}=Y^{\mu}\left(\tau, \sigma^{1}, \sigma^{2}\right)+\xi n^{\mu}\left(\tau, \sigma^{1}, \sigma^{2}\right),
$$

where $x^{\mu}$ are Cartesian, laboratory frame coordinates in Minkowski spacetime, and $\left(n^{\mu}\right)$ is a normalised space-like four-vector, orthogonal to the $\tilde{\Sigma}$ in the covariant sense, i.e.

$$
n_{\mu}\left(\tau, \sigma^{1}, \sigma^{2}\right) Y_{, a}^{\mu}\left(\tau, \sigma^{1}, \sigma^{2}\right)=0, \quad n_{\mu} n^{\mu}=1
$$


where $a=0$ corresponds to $\tau ; a=1, a=2$ correspond to $\sigma^{1}, \sigma^{2} ; Y_{, \tau}^{\mu} \equiv$ $\partial Y^{\mu} / \partial \tau$, etc. The four-vectors $Y_{, \tau}, Y_{, \sigma^{1}}, Y_{, \sigma^{2}}$ are tangent to $\tilde{\Sigma}$. For points lying on the membrane $\xi=0$, and the parameter $\tau$ coincides with the labframe time $x^{0}$. For $\xi \neq 0, \tau$ in general is not equal to the lab-frame time $x^{0}$. Notice that definition (6) implies that $\xi$ is a Lorentz scalar. In the co-moving coordinates the membrane is described by the simple, Lorentz invariant condition $\xi=0$.

The next step is to write Eq.(2) in the new coordinates. It is convenient to introduce extrinsic curvature coefficients $K_{a b}$ and induced metrics $g_{a b}$ on $\tilde{\Sigma}$ :

$$
K_{a b}=n_{\mu} Y_{, a b}^{\mu}, \quad g_{a b}=Y_{, a}^{\mu} Y_{\mu, b},
$$

where $a, b=0,1,2$. The covariant metric tensor in the new coordinates has the following form

$$
\left[G_{\alpha \beta}\right]=\left[\begin{array}{ll}
G_{a b} & 0 \\
0 & 1
\end{array}\right]
$$

where $\alpha, \beta=0,1,2,3 ; \quad \alpha=3$ corresponds to the $\xi$ coordinate; and

$$
G_{a b}=N_{a c} g^{c d} N_{d b}, \quad N_{a c} \equiv g_{a c}-\xi K_{a c} .
$$

Thus, $G_{\xi \xi}=1, G_{\xi a}=0(\mathrm{a}=0,1,2$, as in $(7))$. It follows from formula (10) that

$$
\sqrt{-G}=\sqrt{-g} h\left(\tau, \sigma^{1}, \sigma^{2}, \xi\right),
$$

where as usual $g \equiv \operatorname{det}\left[g_{a b}\right], G \equiv \operatorname{det}\left[G_{\alpha \beta}\right]$, and

$$
h\left(\tau, \sigma^{1}, \sigma^{2}, \xi\right)=1-\xi K_{a}^{a}+\frac{1}{2} \xi^{2}\left(K_{a}^{a} K_{b}^{b}-K_{a}^{b} K_{b}^{a}\right)-\frac{1}{3 !} \xi^{3} \epsilon_{a b c} \epsilon^{d e f} K_{d}^{a} K_{e}^{b} K_{f}^{c} .
$$

Also $g$ can depend on $\tau, \sigma^{1}, \sigma^{2}$. For raising and lowering the latin indices of the extrinsic curvature coefficients we use the induced metric tensors $g^{a b}, g_{a b}$.

The inverse metric tensor $G^{\alpha \beta}$ is given by

$$
\left[G^{\alpha \beta}\right]=\left[\begin{array}{ll}
G^{a b} & 0 \\
0 & 1
\end{array}\right],
$$

where

$$
G^{a b}=\left(N^{-1}\right)^{a c} g_{c d}\left(N^{-1}\right)^{d b}
$$


Explicit formula for $\left(N^{-1}\right)^{a c}$ has the following form:

$$
\begin{aligned}
\left(N^{-1}\right)^{a c}= & \frac{1}{h}\left\{g^{a c}\left[1-\xi K_{b}^{b}+\frac{1}{2} \xi^{2}\left(K_{b}^{b} K_{d}^{d}-K_{b}^{d} K_{d}^{b}\right)\right]\right. \\
& \left.+\xi\left(1-\xi K_{b}^{b}\right) K^{a c}+\xi^{2} K_{d}^{a} K^{d c}\right\}
\end{aligned}
$$

(this is just the matrix inverse to $\left[N_{a b}\right]$; by definition it has upper indices).

In general, the coordinates $\left(\tau, \sigma^{1}, \sigma^{2}, \xi\right)$ are defined locally, in a vicinity of the world-volume $\tilde{\Sigma}$. Roughly speaking, the allowed range of the $\xi$ coordinate is restricted by the smallest of the two curvature radia of the membrane in the local rest frame of a piece of the membrane. We assume that the domain wall is sufficiently narrow so that outside of the region of validity of the co-moving coordinates there are only exponential tails of the domain wall, i.e. the solution is exponentially close to one of the two vacuum solutions. Detailed discussion of the region of validity of the co-moving coordinates has been given in [23].

In the co-moving coordinates the field equation (2) has the following form

$$
\frac{1}{\sqrt{-G}} \frac{\partial}{\partial u^{a}}\left(\sqrt{-G} G^{a b} \frac{\partial \Phi}{\partial u^{b}}\right)+\frac{1}{h} \partial_{\xi}\left(h \partial_{\xi} \Phi\right)-2 \lambda\left(\Phi^{2}-\frac{M^{2}}{4 \lambda}\right) \Phi=0,
$$

where $u^{0} \equiv \tau, u^{1} \equiv \sigma^{1}, u^{2} \equiv \sigma^{2}$.

Let us rescale the scalar field and the $\xi$ coordinate

$$
\Phi\left(x^{\mu}\right) \equiv \frac{M}{2 \sqrt{\lambda}} \phi\left(u^{a}, s\right), \quad \xi \equiv \frac{2}{M} s,
$$

where $\phi\left(u^{a}, s\right)$ and $s$ are dimensionless. Then, equation (16) has the form

$$
\frac{2}{M^{2}} \frac{1}{\sqrt{-G}} \frac{\partial}{\partial u^{a}}\left(\sqrt{-G} G^{a b} \frac{\partial \phi}{\partial u^{b}}\right)+\frac{1}{2} \frac{\partial^{2} \phi}{\partial s^{2}}+\frac{1}{2 h} \frac{\partial h}{\partial s} \frac{\partial \phi}{\partial s}-\left(\phi^{2}-1\right) \phi=0 .
$$

We see that the expansion parameter, i.e. the width $l_{0} \equiv 1 / M$, appears in front of the highest order derivative with respect to $\tau$ present in this equation in such a manner that in the zeroth order approximation the $\tau$ derivative drops out (as well as in the first order approximation in this particular example). It is well-known that in such cases straightforward perturbative expansion in positive powers of the expansion parameter in general is not correct 24]. 
The energy-momentum tensor in our model has the following components

$$
T^{\mu \nu}=\partial^{\mu} \Phi \partial^{\nu} \Phi+\eta^{\mu \nu} L
$$

with $L$ given by formula (1). The laboratory frame energy $E$ is given by the integral

$$
E=\int d^{3} x T^{00}
$$

In this formula the field $\Phi$, which is a scalar with respect to coordinate transformations, can be regarded as a function of the $\left(u^{a}, \xi\right)$ variables. With the help of a formula for differentiation of composite functions $T^{00}$ can be written in the following form

$$
\begin{aligned}
T^{00} & =\left[\left(N^{-1}\right)^{0 a} \Phi_{, a}+n^{0} \Phi_{, \xi}\right]^{2} \\
& +\frac{1}{2} g_{a b}\left(N^{-1}\right)^{a c}\left(N^{-1}\right)^{b d} \Phi_{, c} \Phi_{, d}+\frac{1}{2}\left(\Phi_{, \xi}\right)^{2}+V(\Phi),
\end{aligned}
$$

where $a=0,1,2 ; n^{0}$ is the $\mu=0$ component of the four-vector $\left(n^{\mu}\right)$; the potential

$$
V(\Phi)=\frac{\lambda}{2}\left(\Phi^{2}-\Phi_{0}^{2}\right)^{2}
$$

and the matrix $N^{-1}$ is given by formula (15).

Also the volume element $d^{3} x$ is expressed by the co-moving coordinates,

$$
d^{3} x=\sqrt{-g} \frac{h\left(u^{a}, \xi\right)}{1-\xi K_{0 a} g^{a 0}} d \xi d \sigma^{1} d \sigma^{2} .
$$

Observe that on the hyperplane of constant $x^{0}$ the $\tau$ variable in general becomes a function of $\xi$; this is because

$$
\tau+\xi n^{0}\left(\tau, \sigma^{i}\right)=x^{0}=\text { constant } .
$$

In general, constant $x^{0}$ does not correspond to constant $\tau$, except for the membrane where $\xi=0$, hence $x^{0}=\tau$. This complicates very much calculation of the integral over $\xi$ because in general we do not know the explicit form of the $\tau$ dependence - for this one would have to know explicit solutions of the Nambu-Goto equation for the membrane.

There is a particular case in which the calculation of the energy is relatively simple: when each piece of the membrane associated with the domain wall is at rest at the initial time $x^{0}$. Mathematically, this means that the first derivative of $\vec{Y}$ with respect to $x^{0}$ vanishes. Then, one can show that $n^{0}=0$, hence $x^{0}=\tau$ for all $\xi$. 


\section{Solution by the Hilbert-Chapman-Enskog method}

Following the earlier papers we expand the field $\phi$ in the non-negative powers of $1 / M$

$$
\phi=\phi^{(0)}+\frac{1}{M} \phi^{(1)}+\frac{1}{M^{2}} \phi^{(2)}+\frac{1}{M^{3}} \phi^{(3)}+\ldots,
$$

and we solve equation (18) perturbatively. The expansion parameter is $1 / M$ and not $1 / M^{2}$ because $1 / M$ in the first power is present in the $h$ and $G^{a b}$ functions after passing to the $s$ variable, formulae (17).

Inserting the perturbative Ansatz into equation (18) and expanding the l.h.s. of it in powers of $1 / M$ we obtain the following sequence of equations for $\phi^{(n)}$ :

$$
\begin{gathered}
\frac{1}{2} \partial_{s}^{2} \phi^{(0)}+\phi^{(0)}\left[1-\left(\phi^{(0)}\right)^{2}\right]=0 \\
\frac{1}{2} \partial_{s}^{2} \phi^{(1)}+\left[1-3\left(\phi^{(0)}\right)^{2}\right] \phi^{(1)}=K_{a}^{a} \partial_{s} \phi^{(0)} \\
\frac{1}{2} \partial_{s}^{2} \phi^{(2)}+\left[1-3\left(\phi^{(0)}\right)^{2}\right] \phi^{(2)}=-2 \square^{(3)} \phi^{(0)} \\
+2 K_{a}^{b} K_{b}^{a} s \partial_{s} \phi^{(0)}+3 \phi^{(0)}\left(\phi^{(1)}\right)^{2}+K_{a}^{a} \partial_{s} \phi^{(1)} \\
\frac{1}{2} \partial_{s}^{2} \phi^{(3)}+\left[1-3\left(\phi^{(0)}\right)^{2}\right] \phi^{(3)}=4 K_{c}^{a} K_{a}^{b} K_{b}^{c} s^{2} \partial_{s} \phi^{(0)}-2 \square^{(3)} \phi^{(1)} \\
+2 K_{a}^{b} K_{b}^{a} s \partial_{s} \phi^{(1)}+6 \phi^{(0)} \phi^{(1)} \phi^{(2)}+\left(\phi^{(1)}\right)^{3}+K_{a}^{a} \partial_{s} \phi^{(2)}
\end{gathered}
$$

In these equations

$$
\square^{(3)} \equiv \frac{1}{\sqrt{-g}} \frac{\partial}{\partial u^{a}}\left(\sqrt{-g} g^{a b} \frac{\partial}{\partial u^{b}}\right)
$$

is the three-dimensional d'Alembertian on the world-volume $\tilde{\Sigma}$ of the membrane. Notice that only Eq.(26) is nonlinear. The other equations are linear inhomogeneous ordinary differential equations for $\phi^{(n)}, n \geq 1$.

In general, the range of the variable $s$ in these equations is restricted, $\frac{M}{2} \xi_{-} \leq s \leq \frac{M}{2} \xi_{+}$, because the coordinates $\left(u^{a}, \xi\right)$ do not cover the whole space-time. The values of $\xi_{ \pm}$are found from the condition that $h\left(u^{a}, \xi\right) \geq 0$ for all $\xi$ in the interval $\left(\xi_{+}, \xi_{-}\right)$. In general $\xi_{ \pm}$depend on $u^{a}$. It is clear that 
the function $h$ with generic $K_{a b}$ and $g_{a b}$ can vanish for finite $\xi$, see formula (12). From a mathematical point of view there is no problem - just one has to add more coordinate maps, smoothly matched with the coordinates $\left(u^{a}, \xi\right)$, in order to cover the whole space-time. From a practical, calculational point of view this would be a significant complication with rather little gain in understanding the physics of the not-too-much-curved domain wall we consider in this paper, because the additional coordinate maps would cover the uninteresting from physical point of view region in which the $\Phi$ field is exponentially close to one of the vacuum values. For this reason we do not introduce the additional coordinate maps, and therefore we do not determine the field in the region where the domain wall exponentially merges into the vacuum.

Then another problem arises, namely how to impose the boundary conditions for our solutions, because for finite $\xi=\xi_{ \pm}$the field $\Phi$ in general does not reach the vacuum values. This problem can be circumvented in the following way. We shall find solutions of equations (26-29) assuming that $s$ varies from $-\infty$ to $+\infty$. Notice that these equations formally make sense for all $s$. The solutions have physical meaning only in the restricted range of $s$. Because we presume that the domain wall field for $\xi$ close to $\xi_{ \pm}$is exponentially close to the vacuum values, we require that for large $s$ $\phi^{(0)}$ is exponentially close to \pm 1 , and $\phi^{(n)}, n \geq 1$, are exponentially close to zero. In this manner the boundary conditions at $\xi=\xi_{ \pm}$are fixed uniquely, eventhough in the somewhat implicit manner.

The zeroth order equation (26) has the well-known solutions

$$
\phi_{ \pm}^{(0)}(s)= \pm \text { tanhs } .
$$

They obey the boundary conditions specified above. We take $\phi_{+}^{(0)}$ as the starting point for the approximation procedure. In the case of planar domain wall this $\phi^{(0)}$ coincides with the exact solution (3). The corrections given by $\phi^{(n)}, n \geq 1$, can be regarded as being due to the curvature of the domain wall.

Let us pass to the first order equation (27). On the r.h.s. of it we have the function

$$
\partial_{s} \phi_{+}^{(0)}=\frac{1}{\cosh ^{2} s} .
$$

The crucial step in the Chapman-Hilbert procedure is to observe that the 
operator

$$
\hat{L} \equiv \frac{1}{2} \partial_{s}^{2}+1-3\left(\phi^{(0)}\right)^{2}
$$

present on the l.h.s. of Eqs.(27-29) has a zero eigenvalue. The coresponding eigenfunction coincides with the function (32). (It is identical with a zero mode for a kink in the $1+1$ dimensional version of the considered model (1).) Multiplying the both sides of Eq.(27) by the function (32) and integrating over $s$ in the interval $s \in(-\infty,+\infty)$ we find that the integral on the l.h.s. vanishes, while the r.h.s. is equal to $\frac{4}{3} K_{a}^{a}$. Therefore, we have to accept the consistency condition for our expansion

$$
K_{a}^{a}=0 .
$$

It can be shown that this condition is equivalent to Nambu-Goto equation

$$
\square^{(3)} Y^{\mu}=0
$$

for a relativistic membrane. This is the reason for calling the surface $\tilde{S}$ the membrane. Solutions of the equation (33) for $Y^{i}$ have been discussed in numerous papers, see,e.g., [16, 23]. From Eq.(33) with fixed initial data one can calculate the $Y^{i}$ functions, and subsequently the extrinsic curvature coefficients $K_{a b}$ and the metric $g_{a b}$. Then, from the condition $h>0$ one can determine $\xi_{ \pm}$.

Because of condition (33) the r.h.s. of Eq.(27) in fact vanishes. Then, the general vanishing at the infinity solution of Eq.(27) has the form

$$
\phi^{(1)}=\frac{C\left(u^{a}\right)}{\cosh ^{2} s}
$$

where $C$ does not depend on $s$.

The next equation to be solved is Eq. $(28)$. Because $\phi_{+}^{(0)}$ given by formula (31) does not depend on $u^{a}$, and $K_{a}^{a}=0$, there are simplifications on the r.h.s. of Eq.(28):

$$
\frac{1}{2} \partial_{s}^{2} \phi^{(2)}+\left[1-3\left(\phi^{(0)}\right)^{2}\right] \phi^{(2)}=2 K_{a}^{b} K_{b}^{a} \frac{s}{\cosh ^{2} s}+3 C^{2} \frac{\sinh s}{\cosh ^{5} s} .
$$

Again on the l.h.s. we have the operator $\hat{L}$, however this time the integration with the zero mode (32) gives the identity $0=0$ because the r.h.s. of Eq.(36) is an odd function of $s$. 
General solution of Eq.(36) can be found by a standard technique consisting of first finding two independent solutions of the homogeneous equation $\hat{L} \phi^{(2)}=0$, and next constructing a Green function, see e.g. [26]. As the two linearly independent solutions of the homogeneous counterpart of Eq.(36) we take

$$
\begin{gathered}
\phi_{1}^{(2)}=\frac{1}{\cosh ^{2} s}, \\
\phi_{2}^{(2)}=\frac{1}{8} \sinh (2 s)+\frac{3}{8} \tanh s+\frac{3}{8} \frac{s}{\cosh ^{2} s} .
\end{gathered}
$$

As the Green function we take

$$
G(s, x)=2 \phi_{2}^{(2)}(s) \phi_{1}^{(2)}(x) \Theta(s-x)-2 \phi_{1}^{(2)}(s) \phi_{2}^{(2)}(x)[\Theta(s-x)-\Theta(-x)] .
$$

The general solution of Eq.(36) has the form

$$
\phi^{(2)}=\alpha \phi_{1}^{(2)}+\beta \phi_{2}^{(2)}+\int_{-\infty}^{+\infty} d x G(s, x) f(x)
$$

where $\alpha\left(u^{a}\right), \beta\left(u^{a}\right)$ do not depend on $s$, and

$$
f \equiv 2 K_{a}^{b} K_{b}^{a} \frac{s}{\cosh ^{2} s}+3 C^{2} \frac{\sinh s}{\cosh ^{5} s}
$$

is the r.h.s. of Eq.(36). Formula (39) gives

$$
\phi^{(2)}=c_{1}\left(u^{a}, s\right) \phi_{1}^{(2)}(s)+c_{2}\left(u^{a}, s\right) \phi_{2}^{(2)}(s)
$$

where

$$
\begin{gathered}
c_{1}\left(u^{a}, s\right)=\alpha-6 C^{2} f_{1}(s)-4 K_{a}^{b} K_{b}^{a} f_{2}(s), \\
c_{2}\left(u^{a}, s\right)=\beta-\frac{C^{2}}{\cosh ^{6} s}-4 K_{a}^{b} K_{b}^{a} f_{3}(s),
\end{gathered}
$$

The functions $f_{i}(s)$ are defined by the following formulae

$$
\begin{gathered}
f_{1}(s) \equiv \int_{0}^{s} d x \frac{\sinh x}{\cosh ^{5} x} \phi_{2}^{(2)}(x), f_{2}(s) \equiv \int_{0}^{s} d x \frac{x}{\cosh ^{2} x} \phi_{2}^{(2)}(x), \\
f_{3}(s) \equiv-\int_{-\infty}^{s} d x \frac{x}{\cosh ^{4} x}
\end{gathered}
$$


The functions $f_{1}, f_{2}$ are odd, while $f_{3}$ is even. The function $\phi_{2}^{(2)}$ is given by formula (38). Because this function exponentially grows for $s \rightarrow \pm \infty$, the coefficient function $c_{2}$ has to vanish in this limit. Therefore, we have to put

$$
\beta=0
$$

while $\alpha$ is still arbitrary.

One more restriction comes from the third order equation (29). Integrating it with the zero mode we obtain the following condition

$$
\square^{(3)} C+\left(\frac{\pi^{2}}{4}-1\right) K_{a}^{b} K_{b}^{a} C+\frac{9}{35} C^{3}=\left(\frac{\pi^{2}}{6}-1\right) K_{c}^{a} K_{a}^{b} K_{b}^{c} .
$$

Notice that the function $\alpha$ is not present in (47) - it drops out because an integral giving the coefficient in front of it vanishes. The coefficients in Eq.(47) has been obtained from computations of integrals over $s$.

Condition (47) restricts possible choices of the function $C\left(u^{a}\right)$. It has the form of a non-linear $2+1$ dimensional wave equation. The function $C$ can be regarded as a scalar field on the Nambu-Goto manifold determined from equation (33).

Let us summarize results of our calculations. Up to the second order in $1 / M$

$$
\begin{gathered}
\phi=\tanh s+\frac{1}{M} \frac{C}{\cosh ^{2} s}+\frac{1}{M^{2}} \frac{\alpha}{\cosh ^{2} s} \\
-\frac{1}{M^{2}}\left[C^{2}\left(\frac{6 f_{1}(s)}{\cosh ^{2} s}+\frac{\phi_{2}^{(2)}(s)}{\cosh ^{6} s}\right)+4 K_{a}^{b} K_{b}^{a}\left(\frac{f_{2}(s)}{\cosh ^{2} s}+f_{3}(s) \phi_{2}^{(2)}(s)\right)\right],
\end{gathered}
$$

where $C$ obeys Eq.(47), while for $\alpha$ no explicit restrictions have been obtained till now. However, such restrictions might appear as the consistency condition following from the fourth order equation. Notice that Eq.(47) for the coefficient $C$ introduced in the first order solution (35) has been obtained from the equation (29) two order higher. Thus, in order to claim that formula (48) gives the domain wall solution up to the order $1 / M^{2}$ one should also find $\phi^{(3)}$ and check the consistency condition coming from the fourth order equation.

The fourth order equation has the following form

$$
\frac{1}{2} \partial_{s}^{2} \phi^{(4)}+\left[1-3\left(\phi^{(0)}\right)^{2}\right] \phi^{(3)}=-2 \square^{(3)} \phi^{(2)}-\frac{1}{\sqrt{-g}} \frac{\partial}{\partial u^{a}}\left(\sqrt{-g} K^{a b} \frac{\partial C}{\partial u^{b}}\right) \frac{8 s}{\cosh ^{2} s}
$$




$$
\begin{gathered}
+3 \phi_{+}^{(0)}\left(\phi^{(1)}\right)^{2}+6 \phi_{+}^{(0)} \phi^{(1)} \phi^{(3)}+3\left(\phi^{(1)}\right)^{2} \phi^{(2)} \\
+2 K_{a}^{b} K_{b}^{a} s \partial_{s} \phi^{(2)}+4\left(K_{a}^{b} K_{b}^{a}\right)^{2} s^{3} \partial_{s} \phi_{+}^{(0)}+4 K_{c}^{a} K_{a}^{b} K_{b}^{c} s^{2} \partial_{s} \phi^{(1)} .
\end{gathered}
$$

The r.h.s. of this equation has already been simplified by taking into account the condition (33) and the fact that $\phi_{+}^{(0)}$ does not depend on $u^{a} . \phi^{(3)}$ is given by a formula analogous to (39). Repeating once again the calculation leading to the consistency conditions we obtain from Eq.(49) the following condition

$$
\square^{(3)} \alpha+\frac{1}{2} K_{a}^{b} K_{b}^{a} \alpha-\frac{36}{35} C^{2} \alpha=0 .
$$

Thus, the fourth order equation (49) gives the restriction (50) for $\alpha$.

The final step in our procedure is to make a convenient choice of the initial position and velocities of points of the membrane. Its evolution at later times is then determined by the Nambu-Goto equation (33). Let us recall that the membrane is the auxilliary object merely, used to define the co-moving coordinate system, so that the choice of the initial data has no physical meaning. The most natural choice is that at the initial value of $\tau$ the position of the membrane as well as its velocities coincide with those of the core. By the definition of the membrane, for its points $s=0$. On the other hand, we find from formula (48) that for the core (i.e. for the points where $\phi$ vanishes)

$$
s=s_{0} \equiv-\frac{C}{M}-\frac{\alpha}{M^{2}}+\mathcal{O}\left(M^{-3}\right) .
$$

Therefore, our choice of the initial data for the membrane means that $s_{0}=$ $0, \partial_{\tau} s_{0}=0$ at the initial value of $\tau=\tau_{0}$. This implies that

$$
\begin{gathered}
C\left(u^{a}, \tau_{0}\right)=0, \quad \frac{\partial C}{\partial \tau}\left(u^{a}, \tau_{0}\right)=0, \\
\alpha\left(u^{a}, \tau\right)=0, \quad \frac{\partial \alpha}{\partial \tau}\left(u^{a}, \tau_{0}\right)=0 .
\end{gathered}
$$

Notice that the more general solution $\alpha=-M C$ is not acceptable, because one should not mix different orders of the perturbative expansion. With the initial conditions (53) equation (50) implies that

$$
\alpha=0
$$


for all $\tau$. On the other hand, the term on the r.h.s. of equation (47) acts as an external, curvature dependent force which in general leads to nontrivial $\tau$-dependence of the function $C$ even for the trivial initial data (52).

Thus, the domain wall solution constructed to the second order in $1 / M$ with the Hilbert-Chapman-Enskog method has the form (48) with $\alpha=0$ and $C$ determined from the equation (47) with the initial data (52).

The formula (51) gives the one-to-one mapping between the core and the membrane: the point $\left(u^{a}, s=0\right)$ of the membrane is mapped to the point $\left(u^{a}, s=s_{0}\right)$ of the core.

\section{$4 \quad$ Examples}

In this Section we specify our general solution to describe simple, symmetrical domain walls.

(a) The planar domain wall. Let us start from the simplest domain wall: the planar, static one. In our formalism this means that the membrane is a motionless plane. In this case $K_{a b}=0$, so that the external force in Eq.(47) vanishes. Therefore $C=0$, and the solution (48) coincides with the exact solution (3). Energy per unit square of the membrane is equal to

$$
\frac{E}{|\tilde{S}|}=\frac{2}{3} M \Phi_{0}^{2}
$$

where $|\tilde{S}|$ denotes the area of a piece of the membrane.

(b) The cylindrical domain wall. Now the membrane is a cylinder of the radius $r$. We assume that the cylinder does not move as a whole. Using the formulae of the Section 2 one can easily calculate the extrinsic curvatures $K_{a b}$ and the metrics $g_{a b}$. The Nambu-Goto equation has the following form

$$
\frac{\ddot{r}}{1-\dot{r}^{2}}+\frac{1}{r}=0
$$

where the dots denote derivatives with respect to $\tau$. This equation has the "energy" first integral given by the formula

$$
E^{N-G}=\frac{r}{\sqrt{1-\dot{r}^{2}}}
$$


where $E^{N-G}$ is a constant. We also find that

$$
K_{b}^{a} K_{a}^{b}=\frac{2}{r^{2}\left(1-\dot{r}^{2}\right)}=\frac{2\left(E^{N-G}\right)^{2}}{r^{4}}, \quad K_{c}^{a} K_{a}^{b} K_{b}^{c}=0 .
$$

Thus, the external force in the equation (47) vanishes, and the membrane coincides with the core for all times - the zeros of the scalar field follow the Nambu-Goto trajectory.

The solution $\phi$ is given by formula (48) with $C=\alpha=0$ and $K_{b}^{a} K_{a}^{b}$ as in the first of formulae (56).

The energy for the cylindrical domain wall solution is also easily calculated with the help of formulae $(20),(21),(23)$. Because of the difficulty mentioned at the end of Section 2, we calculate the energy at the initial $\tau=\tau_{0}$, assuming that the core is then at instant rest. Thus,

$$
r\left(\tau_{0}\right)=\dot{r}\left(\tau_{0}\right)=0,
$$

and $C$ has the initial value (52). At later time

$$
r(\tau)=r_{0} \cos \frac{\tau}{r_{0}}
$$

as it follows from Eq.(55). It is easy to see that $E^{N-G}=r_{0}$.

Let us notice here that our formulae are applicable only for $\tau / r_{0} \in$ $[0, \pi / 2-\delta]$, with some $\delta>0$. The reason is that for $\tau / r_{0}$ approaching $\pi / 2$ it no longer true that on the inner side of the domain wall the field $\Phi$ is exponentially close to the vacuum. The value of $\delta$ can be estimated analogously as in the first of papers 23.

The result for the energy per unit square of the membrane is the same as for the planar domain wall. Thus, the extrinsic curvature of the cylinder does not influence the total energy per unit square of the membrane (to the order $\left.1 / M^{2}\right)$. However, the density of energy across the domain wall is changed in comparison with the planar case. Its maximum still lies on the core, but it is flattened: the maximal value of the energy density is equal to

$$
\frac{M}{2} \Phi_{0}^{2}\left(1-\frac{8 f_{3}(0)}{M^{2} r_{0}^{2}}\right)
$$

where $f_{3}(0)=\frac{2}{3} \ln 2-\frac{1}{6} \approx 0.30$, and $r_{0}$ is the initial radius. 
(c) The spherical domain wall The membrane is a sphere of the radius $r$. The Nambu-Goto equation has the following form

$$
\frac{\ddot{r}}{1-\dot{r}^{2}}+\frac{2}{r}=0 \text {. }
$$

Its solutions have been considered in, e.g., [16, 23]. $r(\tau)$ is given by an elliptic function and it is oscillating with $\tau$. The "energy" integral is given by the formula

$$
E^{N-G}=\frac{r^{2}}{\sqrt{1-\dot{r}^{2}}},
$$

where $E^{N-G}$ is a constant. Again we consider evolution of the domain wall only in a part of the first quarter of the cycle of the oscillations predicted by Eq.(57), before the spherical domain wall collapses to the center.

For $K_{b}^{a} K_{a}^{b}$ we have the formula

$$
K_{b}^{a} K_{a}^{b}=\frac{6}{r^{2}\left(1-\dot{r}^{2}\right)}=\frac{6\left(E^{N-G}\right)^{2}}{r^{6}} .
$$

The external force in Eq.(47) does not vanish:

$$
K_{c}^{a} K_{a}^{b} K_{b}^{c}=\frac{6}{r^{3}\left(1-\dot{r}^{2}\right)^{3 / 2}}=\frac{6\left(E^{N-G}\right)^{3}}{r^{6}} .
$$

Formulae (58), (59) have been obtained by taking $u^{1}=\phi, u^{2}=\theta$, where $\phi, \theta$ are the usual azimuthal and polar angles parametrising the spherical membrane. The vector $\vec{n}$ has been directed to the outside of the sphere. Then $s>0(s<0)$ corresponds to the outside (inside) of the spherical membrane.

Equation (47) has now the following form

$$
\ddot{C}-6\left(\frac{\pi^{2}}{4}-1\right) \frac{C}{r^{2}}-\frac{9}{35}\left(1-\dot{r}^{2}\right) C^{3}=-\frac{\pi^{2}-6}{r^{3} \sqrt{1-\dot{r}^{2}}} .
$$

At the initial instant $\tau=\tau_{0}: C=0, r=r_{0}, \dot{r}=0$, and the acceleration $\ddot{C}=-\left(\pi^{2}-6\right) / r_{0}^{3}<0$. Thus, for $\tau>0 C$ will become more and more negative. It follows from formula (51) that for the core $s=s_{0}=-C / M>0$. This means that the core lags behind the Nambu-Goto membrane. 
For the total energy per unit square of the membrane for the spherical domain wall we obtain a positive correction due to the curvature of the sphere

$$
\frac{E}{4 \pi r_{0}^{2}}=\frac{2}{3} M \Phi_{0}^{2}\left(1+\frac{3 d_{0}}{M^{2} r_{0}^{2}}\right)
$$

where

$$
d_{0} \equiv \int_{-\infty}^{+\infty} \frac{s^{2}}{\cosh ^{4} s} d s=\frac{\pi^{2}}{9}-\frac{2}{3} \approx 0.43
$$

As usual, we have calculated the energy at the initial instant; then $r=r_{0}, \dot{r}=$ $0 ; C=0, \partial_{\tau} C=0$. We see that the total energy $E$ differs from the zeroth order result by the constant term $8 \pi d_{0} \Phi_{0}^{2} / M$. The maximum of the energy density at the initial instant lies on the core, and the maximal value of the energy density is equal to

$$
\frac{M}{2} \Phi_{0}^{2}\left(1-\frac{24 f_{3}(0)}{M^{2} r_{0}^{2}}\right)
$$

Thus, the energy density is more flattened than in the case of cylindrical domain wall.

The facts that the core shrinks more slowly than the Nambu-Goto membrane, and that the energy per unit square has the positive correction increasing with the curvature, mean that the spherical domain wall exhibits an effective rigidity.

\section{$5 \quad$ Remarks}

(a) In our opinion, the main advantage of the method proposed in this paper is that it yields the domain wall solution without invoking the effective action. In this manner we avoid many problems of the effective action method, e.g. handling a model with higher derivatives. Another advantage is that the comoving coordinate system is tied always to the Nambu-Goto type membrane. Equations of motion for this type of membrane are probably the simplest ones to be dealt with.

In comparison with the polynomial approximation, [23], in which one approximates the field inside the domain wall by a simple polynomial, the present approach has the advantage that it is based on the expansion with respect to the clearly defined parameter, i.e. the width. Therefore, it is easy 
to point out the limit in which the zeroth order solution is more and more accurate: $C / M$ and $K_{b}^{a} K_{a}^{b} / M^{2}$ tending to zero. The polynomial solution never is exact.

(b) The corrections $\phi^{(1)}, \phi^{(2)}$ to the zeroth order solution do not change significantly the picture of the evolution of the domain wall obtained from the zeroth order solution. For example, it is easy to see that in the case of spherical domain wall the difference between the radia of the core and of the membrane is of the order $l_{0}^{2} / r_{0}$ - it is smaller than the width of the domain wall. Nevertheless, it is nice to know how to construct the more accurate solution. Moreover, there are questions which one cannot answer without detailed knowledge of the internal structure of the domain wall. Let us mention here the problem of excitations of the domain wall. They can be regarded as bound states of certain particles with the domain wall. In order to calculate spectrum of such excitations one has to know details of the shape of the scalar field inside of the domain wall.

(c) In the present paper we have completely neglected the possibility that the field $\Phi$ can have a component oscillating with the frequency of the order $M$. Because $M$ is the mass of the scalar particle present in the nontopological sector of the model (1), one should expect that such component is in general present. It could be regarded as describing an emission or absorption of the scalar particles by the domain wall. Such oscillating component can be calculated in an extended perturbative expansion, which could be constructed, e.g., by generalising considerations of Section III of the first of papers 23.

The problem of excitations of the domain wall and the problem of radiation probably deserve a separate investigation.

\section{References}

[1] See, e.g.,M.Baker,J.S.Ball and F.Zachariasen, Phys.Rep. 209, 73 (1991); C.Olson, M.G.Olsson and K.Williams, Phys.Rev. D45, 4307 (1992).

[2] T.W.B.Kibble, J.Phys. A99, 1387 (1976); A.L.Vilenkin, Phys. Rep. 121, 263 (1985).

[3] See, e.g., J.Slonczewski, in Physics of Defects (Les Houches Session XXXV, 1980). North-Holland Publ. Comp., Amsterdam, 1981. 
[4] R.P.Huebener, Magnetic Flux Structures in Superconductors. SpringerVerlag,Berlin - Heidelberg - New-York, 1979.

[5] R.J.Donnelly, Quantized Vortices in HeliumII. Cambridge University Press, Cambridge, 1991.

[6] S.Chandrasekhar and G.S.Ranganath, Adv. Phys. 푸, 507 (1986).

[7] H.B.Nielsen and P.Olesen, Nucl.Phys. B61, 45 (1973).

[8] For a review of an early numerical work see V.G. Makhankov, Phys.Rep. 35, 1 (1978). Chapt.7.

[9] E.P.S.Shellard, Nucl.Phys.ㄹ283, 624 (1987).

[10] L.M.Widrow, Phys.Rev.D40, 1002 (1989).

[11] D.Förster, Nucl.Phys. B B81, 84 (1974).

[12] J.-L.Gervais and B.Sakita, Nucl.Phys. B91, 301 (1975).

[13] N.Turok and K.Maeda, Phys.Lett.B202, 376 (1988).

[14] R.Gregory, Phys.Lett.ㄹ206, 199 (1988).

[15] S.M.Barr and D.Hochberg, Phys.Rev.D39, 2308 (1989).

[16] R.Gregory, D.Haws and D.Garfinkle, Phys.Rev. D42, 343 (1990).

[17] V.Silveira and M.D.Maia, Phys.Lett.A174, 280 (1993).

[18] A.L.Larsen, Phys.Lett.A181, 369 (1993).

[19] B.Carter and R.Gregory, preprint DAMTP/R-94/41 (hep-th/9410095).

[20] H.Arodź and P.Wȩgrzyn, Phys.Lett.ㄹ291, 251 (1992).

[21] V.Silveira, Phys.Rev. D41, 1914 (1990).

[22] Uri Ben-Ya'acov, Nucl.Phys. B382, 597 (1992).

[23] H.Arodź and A.L. Larsen, Phys.Rev. D49, 4154 (1994); H.Arodź, preprint TPJU-29/94 (hep-th/9501073). 
[24] See, e.g., E.J.Hinch, Perturbation Methods. Cambridge University Press, Cambridge, England, 1991.

[25] N.G.van Kampen, Stochastic Processes in Physics and Chemistry. North-Holland Publ.Comp., Amsterdam, 1987. Chapt.8,§7.

[26] G.A. Korn and T.M. Korn, Mathematical Handbook. IInd Edition. McGraw-Hill Book Comp., New York, 1968. Chapt.9.3-3. 